\title{
Basic services for the management of Virtual Enterprises - A Case Study
}

\author{
L. M. Spinosa ${ }^{1}$, A. C. M. Hofmann ${ }^{2}$, R. J. Rabelo ${ }^{1}$ and A. A. Pereira \\ Klen ${ }^{1}$
}

${ }^{1}$ G-SIGMA - Intelligent Manufacturing Systems Group, Mechanical Engineering Department of the Federal University of Santa Catarina, Brazil, Tel. (55) 48 3319387, Fax: (55) 48 2341519,E-mail: spinosa@gsigma-grucon.ufsc.br; rabelo@, gsigma-grucon.ufsc.br; klen@gsigma-grucon.ufsc.br; ${ }^{2}$ Akros/Herten Company, Tel.: (55) 47 4363811, Fax: (55) 47 4360901,E-mail: ahofmann@akros.com.br

\begin{abstract}
The main contribution of this work lies in giving rise and triggering further research towards the development of software dedicated to the VE management, since this is just an attempt to give direction to a complex problem. This paper introduces a model describing a basic set of services aiming to support the management of important situations arising during the creation and operation phases of VEs in manufacturing contexts. The model contemplates a study of some cases related with the supply chain of Akros company. All this work has been motivated by the Esprit project Prodnet-II.
\end{abstract}

\author{
Keywords \\ Virtual enterprise, case study, supply chain management
}




\section{INTRODUCTION}

The application of the Virtual Enterprise (VE) concept by industries has been seen as a powerful strategic action in order to face the challenges of a global economy (Browne et al., 1995). In this context, the improvement of the supply-chain management (SCM) is viewed as a must nowadays. This paper aims at introducing a model, which address some basic services related with SCM in order to conduct the creation and operation phases of a VE. Initially, the Akros supply-chain is presented in order to introduce the whole scenario in which the basic services were identified. Afterwards, four cases composing this scenario are analysed in order to identify the main end-user's requirements and the main information exchanged among the VE partners. Finally, based on the identified requirements, the basic services are presented and conclusive remarks are made concerning the improvements and limitations of this work.

This research has been developed in the scope of Prodnet-II Esprit project (Production Planning and Management in an Extended Enterprise) (Camarinha-Matos et al., 1997) which is supported by the European Union and by the CNPq (the Brazilian Council for Technological Development and Research). Prodnet-II aims at designing and developing an open platform and adequate IT protocols and mechanisms to support virtual enterprises.

\section{THE AKROS SUPPLY-CHAIN}

Akros Group is composed of several complementary and autonomous companies specialised on the production of pipes, sanitary accessories and moulds for the plastic injection industry. The current main interest of Akros is to improve its competitiveness in order to respond to an even more global manufacturing and to the Brazilian market opening policies. In this sense, Akros is aware that VEs can be created based on their interest to link its suppliers, its customers and its own companies in a whole supplychain. Each member of this network should be extended with software components to support the information exchange. The members, however, do not perform the whole end product in isolated facilities, but rather they operate as cooperative nodes (Figure 1). In this sense, one of the members becomes the "co-ordinator» being the one responsible for the VE operation.

This scenario characterizes a complex decentralized decision-making process and may strongly impact the management methods of the involved enterprises. This represents one of the most important changes and operational challenges that Akros has to deal with, and, therefore, it is fundamental to understand the information flow between the different VE members. The cases illustrated bellow present a first attempt to identify that flow. 


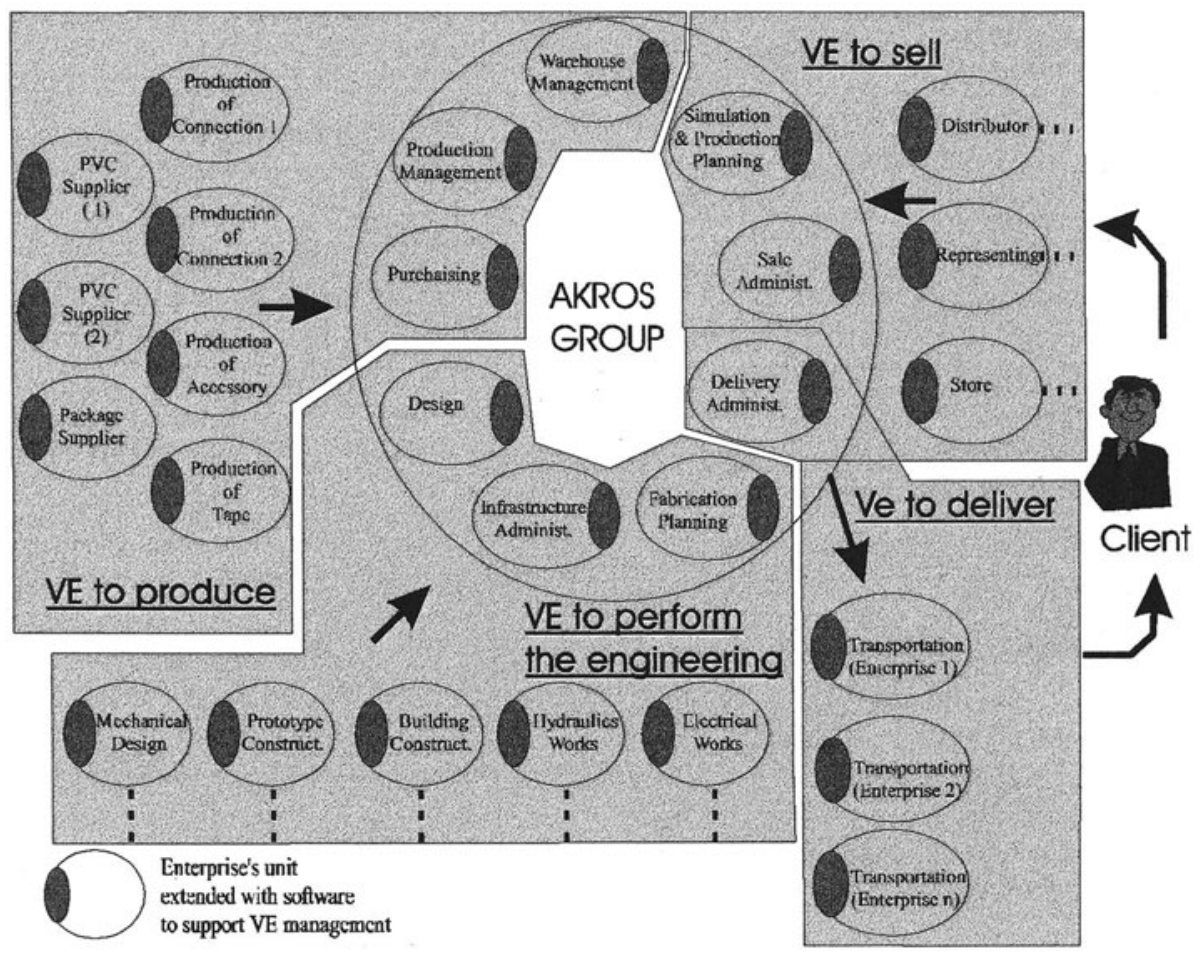

Figure 1 - The Akros supply-chain. The focus is on processes related to the VE creation and operation.

\subsection{A VE to sell products}

The involved members inside Akros are: Sale Administration Unit, Delivery Administration Unit, as well as Simulation and Production Planning Unit. Outside Akros the following members can be identified: distributors, representatives and specialised stores. The Sale Administration Unit co-ordinates the VE. Figure 2 shows the basic information flow involved with the management of such a VE.

\subsection{A VE to perform the engineering of products}

This VE executes, in a co-ordinate way, the engineering of new products or the modification of an existing one. The members inside Akros are: Production Planning Unit, Design Unit and Infrastructure Administration Unit. Outside Akros specialised enterprises on mechanical design, construction of prototypes, buildings, electrical and hydraulics plants are involved. The Design Unit co-ordinates the VE. The basic information flow involved with the management of the present VE is shown in Figure 3. 


\begin{tabular}{c|l}
\hline Arc & information (data) \\
\hline 1 & Stock level \\
\hline 2 & Orders issued from small levels of stock \\
\hline 3 & Sales indicators \\
\hline 4 & Orders issued from sales indicators \\
\hline 5 & Orders \\
\hline 6 & Status orders \\
\hline 7 & Orders to be simulated \\
\hline 8 & Results of order simulation \\
\hline 9 & Request to deliver products \\
\hline 10 & Stalus of requests to deliver products \\
\hline 11 & Marketing indicators \\
\hline
\end{tabular}

Figure 2 - Basic information flow to manage the VE to sell the Akros products.

\begin{tabular}{c|l|l|l}
\hline Arc & information (data) & 12 & Specifications to hydraulics plants \\
\hline 1 & Needs on new products or change of older ones & 13 & Status of hydraulics plants \\
\hline 2 & Technical Projects & 14 & Specifications to electrical plants \\
\hline 3 & Needs on changes & 15 & Status of electrical plants \\
\hline 4 & Fabrication specifications to industrial installation \\
\hline 5 & Project specifications to industrial installation \\
\hline 6 & Project specifications to prototype construction \\
\hline 7 & Status of prototype construction \\
\hline 8 & Project specifications to mechanical design \\
\hline 9 & Status of mechanical design \\
\hline 10 & Specifications to construct the buildings \\
\hline 11 & Status of building constructions \\
\hline &
\end{tabular}

Figure 3 - Basic information flow to manage the VE to perform the engineering of products.

\subsection{A VE to produce}

This case concerns a VE to produce one kind of accessory, two kinds of connections and one kind of tape. Inside Akros the following members have been identified: Production Management Unit (the VE co-ordinator), Purchasing Unit and Warehouse Management Unit. Outside Akros the members are: three PVC material and package suppliers, and four production sites for connections, accessory and tape. The basic information flow involved with the management of such a VE is shown in the Figure 4. 


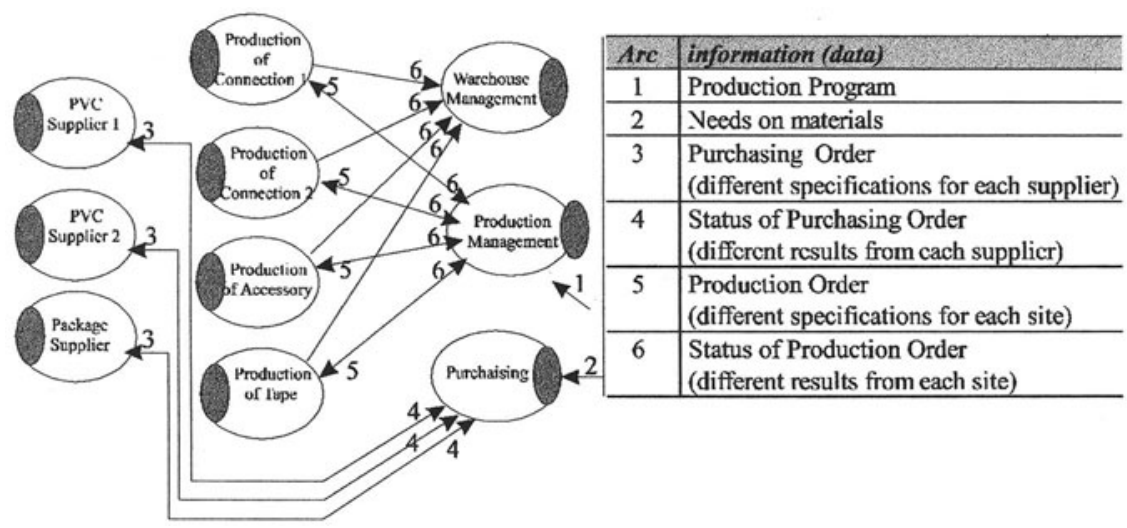

Figure 4-Basic information flow to manage the VE to produce some products.

\subsection{A VE to deliver}

The main involved unit inside Akros is Delivery Unit (the VE co-ordinator). Outside Akros there is only one kind of partner i.e. the transport enterprises. Figure 5 shows the information flow related with the VE management.

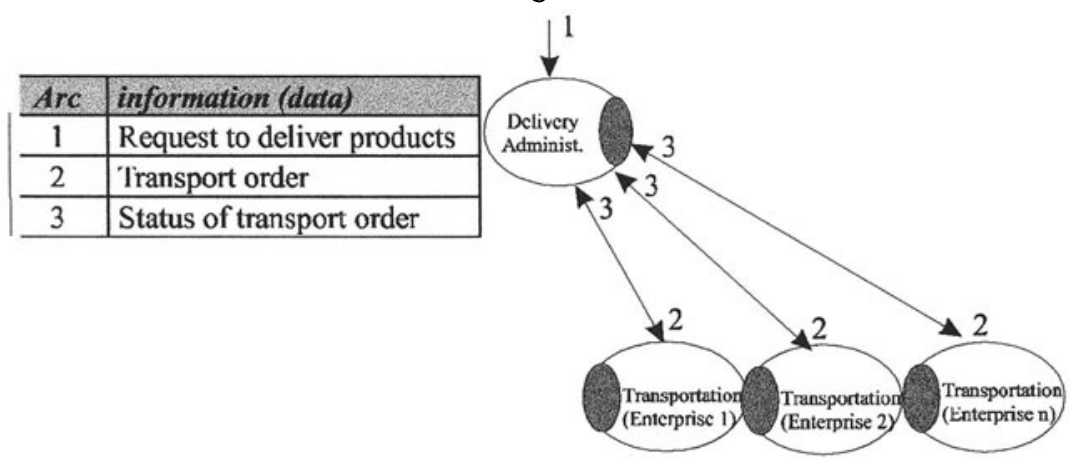

Figure 5 - Basic information flow to manage the VE to deliver.

\section{THE VE MANAGEMENT MODEL - BASIC SERVICES}

The analysis of the above cases led us to conclude that in order to create and operate the VEs it is necessary the execution, by the VE members, of a set of precise actions to guide and control the information flow previously described. These actions can be mainly accomplished if a set of management services is provided. Following, a first attempt of an organised set of basic management services is presented. In this sense, a model has been defined and represented with IDEF0 diagrams (IDEF0, 1993), which are mainly used to represent functional aspects of several kinds of domains. The main notion besides IDEF0 diagrams is that a function is activated by means of a control event and transforms an input information in an output information through the use of precise means (Figure 6). As far as the Basic VE Management services are concerned, the IDEF0 diagrams give rise to the identification of the logical sequence of their 
execution, the information flow between them and some software able to support them as well.

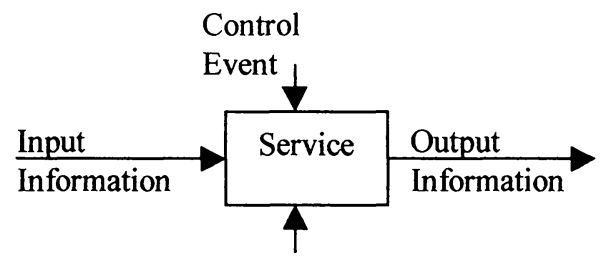

Software

Figure 6 - IDEF0 inputs and outputs.

As suggested in the Figure 7, a well-defined hierarchy (decomposition) of services has been observed,. In order to simplify the introduction of the model, only the filled boxes of the diagram are further detailed.

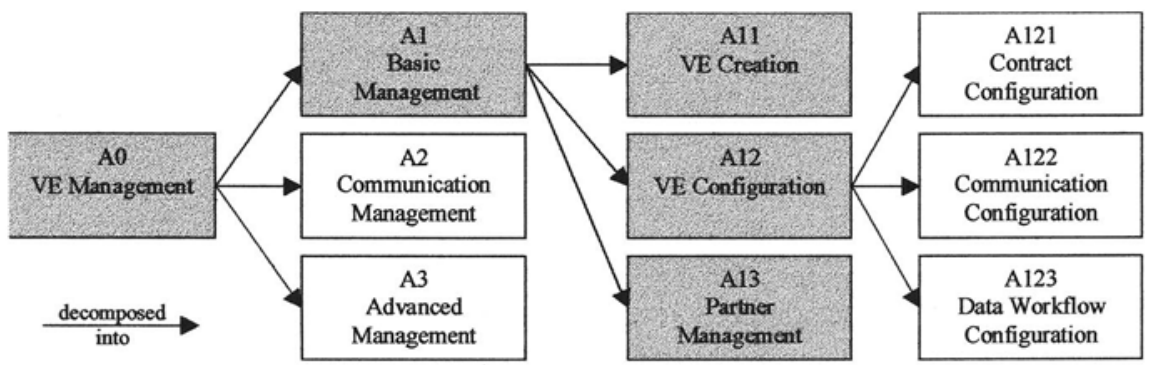

Figure 7 - Main diagrams composing the VE Management Model with focus on Basic Management Services.

\section{AO - VE Management (see Figure 8)}

This is the root of the model. It represents the entire set of services able to achieve the Management of Virtual Enterprises. The services are organised in three main groups: Basic Management (A1), Communication Management (A2) and Advanced Management (A3). The Advanced Management can be viewed as highly evolved services comprising several aspects mainly related with the VE logistics and advanced decision-making processes. The Communication Management considers all relevant services necessary to establish communication between any member of the VE. The Basic Management group, as the central point of this paper, is further detailed.

\section{Al - Basic Management services (see Figure 9)}

This group of services faces the main requirements identified by the case studies of Akros. It corresponds to precise management services as shown by the following subgroups:

- VE creation (A11) - It identifies a set of potential members and establishes with them basic engagements to participate in a VE. At the end of this task, the VE is created and a Creation Plan (script) containing information about the members is generated. 
- VE Configuration (A12) - Based on the Creation Plan a set of configuration services is carried out to define initial conditions for the operation of the VE. The whole information concerning the configuration aspects are put together in a Configuration Plan (script).

- Partner Management (A13) - Based on the Configuration Plan, a set of actions aiming to manage the VE partners is "executed" in an automatic way. Some of them are the generation of production orders according to a pre-defined frequency, the reception of information sent by the members, and the application of rules defining the data workflow of the VE.

\section{A11 - VE Creation services (see Figure 9)}

The following sub-groups compose the VE creation services:

- Announcement of needs (A111) - This service specifies and sends in an automatic way an announcement to potential VE members. An example of the announcement could be: product or service requested ("PVC", "plastic packing", etc.), product or service details, deadline of deliveries; requirements to be a member, and so on.

- Partner Information Retrieval (A112) - Once the deadline to answer the announcement has been expired, this service provides a list of interested members.

- Partner Evaluation (A113) - According to the answers, a sort of implied members is done taking some parameters into account. Such parameters are based on requirements defined in the announcement. The most interesting members are then selected.

- Partners Classifying (A114) - The selected members are catalogued in a database.

- Contract Definition (A115) - This service specifies and sends a contract to the catalogued suppliers. At this level, the contract only takes the formation of possible VEs into account, and does not represent the engagement to execute a service or provide a product.

\section{A12 - VE Configuration services (see Figure 8)}

This group of services is decomposed into the following sub-groups:

- Contract Configuration (A121) - This service details the VE contract to each selected member. Additional information is also taken into account, for instance: the payment details, the quality parameters of the product/service, the logistics parameters of the production/ execution of services, and the punishment actions when the preceding parameters are not respected.

- Communication Configuration (A122) - Several communication aspects are specified in this service such as those for the information exchange (Security protocol, STEP, EDI or Prodnet self protocol), those for sending orders (quantity, colour, project of the product/service), and those for feedback (order status, quality status, product/service status).

- Data Workflow Configuration (A123) - This service defines a set of rules expressed by single sentences like IF A THEN B, which guide the paths of data among the members. For example: "IF (order was sent to member) THEN (inform Management of Invoices Unit) ", "IF (quality parameter is under the limits) THEN (inform Management of Logistics and send a fax to member). 


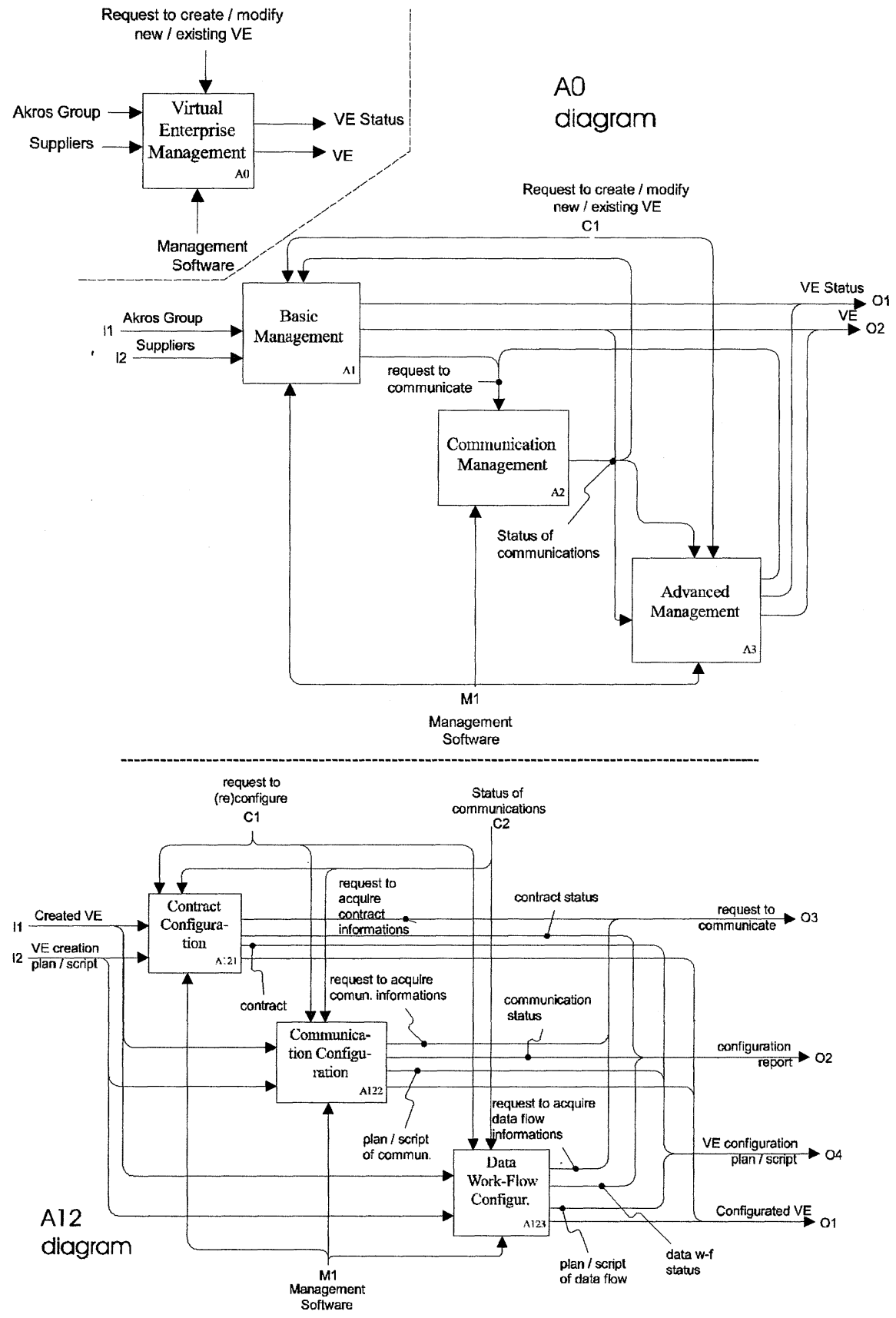

Figure $8-\mathrm{A} 0$ and $\mathrm{A} 12$ diagrams. 


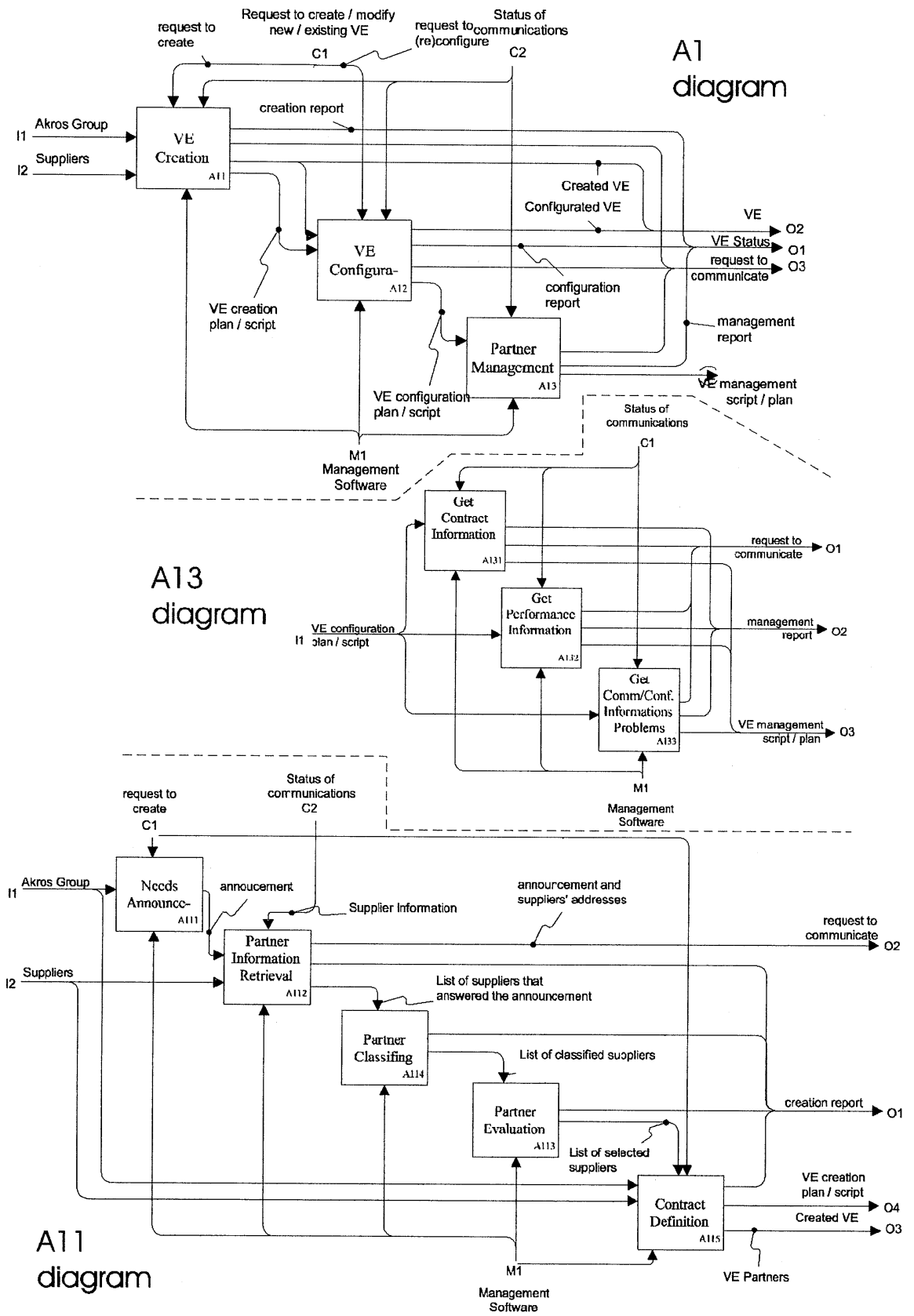

Figure $9-\mathrm{A} 1, \mathrm{~A} 11$ and A13 diagrams. 


\section{A13 - Partner Management services (see Figure 9)}

The following sub-groups compose the VE creation services:

- Get Contract Information (A131) - Based on the VE configuration, the members are requested to send the necessary information to compose the VE contract.

- Get Performance Information (A132) - According to a frequency defined in the previous contract, a request is sent to the members to provide information such as quality and logistics parameters of the production.

- Get Problems (A133) - This group of services represents a "channel" to the members to inform problems such as those related with the communication means and the configuration settings.

\section{CONCLUSIONS}

This paper presents some aspects related to the management of Virtual Manufacturing Enterprises with the aid of the identification of some basic services. By means of a join effort with an end-user (Akros Group), a list of these services was proposed taking into account four different cases of VE creation and operation.

This first attempt to suggest some services was related to the information flow modelling, which is not complete at all. It will be completed along the Prodnet-II project execution regarding both the Akros case and others.

The main contribution of this work lies in giving rise and triggering further research towards the development of software dedicated to the VE management, since this is just an attempt to give direction to a complex problem. Also, the proposed model helps Akros to realize and face some important changes and operational challenges necessary to the adoption of VE concepts, which claim for precise organisational methods as well as specialised profile of the workers.

\section{ACKNOWLEDGMENTS}

The authors would like to thank the CNPq (The Brazilian Council for Technological Development and Research) and the European Union for the financial support for this work.

\section{REFERENCES}

Browne, J.; Sackett, P.; Wortmann, J., Future manufacturing systems: Towards the extended enterprise. Computer in Industry, Special Issue on CIM in the Extended Enterprise, 25 N 3, 235-254, 1995.

Camarinha-Matos, L., Lima, C., Osorio, A. L., The PRODNET platform for production planning and management in virtual enterprises. Proc. Of the $4^{\text {th }}$ International Conference on Concurrent Enterprising, 8-10 October 1997, Nottinghan, UK.

IDEFØ as a standard for Function Modelling in FIPS Publication 183, in Computer Systems Laboratory of the National Institute of Standards and Technology (NIST), December 1993. 